\title{
An Analytical Hierarchy Process Combined with Game Theory for Interface Selection in $5 \mathrm{G}$ Heterogeneous Networks
}

\author{
Mostafa Zaman Chowdhury ${ }^{1,2}$, Md. Tashikur Rahman', and Yeong Min Jang ${ }^{1, *}$ \\ ${ }^{1}$ Dept. of Electronics Engineering, Kookmin University \\ Seoul 02707, Korea \\ [e-mail: mzaman@kookmin.ac.kr, yjang@kookmin.ac.kr] \\ ${ }^{2}$ Dept. of Electrical and Electronic Engineering, Khulna University of Engineering \& Technology \\ Khulna 9203, Bangladesh \\ [e-mail: rahmantashik@gmail.com] \\ *Corresponding author: Yeong Min Jang
}

Received July 24, 2018; revised April 8, 2019; revised July 22, 2019; revised August 19, 2019; accepted September 16, 2019; published April 30, 2020

\begin{abstract}
Network convergence is considered as one of the key solutions to the problem of achieving future high-capacity and reliable communications. This approach overcomes the limitations of separate wireless technologies. Efficient interface selection is one of the most important issues in convergence networks. This paper solves the problem faced by users of selecting the most appropriate interface in the heterogeneous radio-access network (RAN) environment. Our proposed scheme combines a hierarchical evaluation of networks and game theory to solve the network-selection problem. Instead, of considering a fixed weight system while ranking the networks, the proposed scheme considers the service requirements, as well as static and dynamic network attributes. The best network is selected for a particular service request. To establish a hierarchy among the network-evaluation criteria for service requests, an analytical hierarchy process (AHP) is used. To determine the optimum network selection, the network hierarchy is combined with game theory. AHP attains the network hierarchy. The weights of different access networks for a service are calculated. It is performed by combining AHP scores considering user's experienced static network attributes and dynamic radio parameters. This paper provides a strategic game. In this game, the network scores of service requests for various RANs and the user's willingness to pay for these services are used to model a network-versus-user game. The Nash equilibria signify those access networks that are chosen by individual user and result maximum payoff. The examples for the interface selection illustrate the effectiveness of the proposed scheme.
\end{abstract}

Keywords: AHP, game theory, network-selection, network attributes, Nash equilibrium, payoff, RANs, strategic game.

A preliminary version of this paper appeared in ICTC 2016, Oct. 19-21, Jeju Island, Korea. This version includes a detail extension in terms of system analysis and supporting results for interface selection. This research was supported by the MSIT (Ministry of Science and ICT), Korea, under the ITRC (Information Technology Research Center) support program (IITP-2018-0-01396) supervised by the IITP (Institute for Information \& communications Technology Promotion) and the Korea Research Fellowship Program (2016H1D3A1938180). 


\section{Introduction}

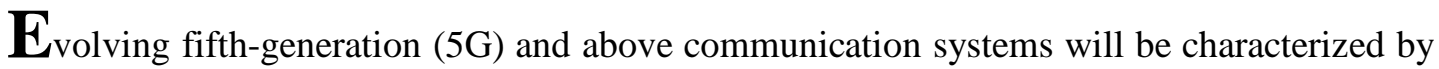
massive device connectivity, ultra-high data rates, ultra-high security, ultra-low latency, and ultra-low energy consumption. Recently, wireless networks have seen incredible increase in the numbers of subscribers as well as in the diversity of traffic. According to a CISCO data analysis [1], the typical mobile data rate will exceed 20 Mbps in 2021 and traffic will exceed half zettabyte per year. These data will be served through heterogeneous networks (HetNets). To facilitate this radical growth in users and service types, we have realized the convergence of HetNets by which numerous radio-access-technologies (RATs) coexist. This convergence has emerged as a key technique for achieving 5G-and-beyond systems and consists not only of the convergence of radio frequency (RF) technologies but also RF and optical wireless communication (OWC) technologies. The optical band will provide emerging solution for the development of upcoming high-capacity OWC networks. Future networks will adopt multi-tier network architectures including satellite, macrocells, small cells, attocells, and relays. In future, 5G and above communication systems will use "network of networks, instead of a single access network. To achieve such goal, it will necessiate seamless integration among diverse kinds of networks in such a way that HetNets collaborate each other. Efficient interface selection is one of the most important issues in convergence networks. Fig. 1 shows an illustrative example of the network-selection problem. Various access networks, e.g., macrocellular BS (MBS), mobile worldwide interoperability for microwave access (WiMAX), small-cell base station (sBS) [2, 3], wireless fidelity (WiFi), and numerous optical networks, are available to serve users. According to various criteria, an optimal network interface is selected to provide a specific type of service to a user. The OWC networks [4-7] include visible-light communication, optical-camera communication, and light fidelity (LiFi).

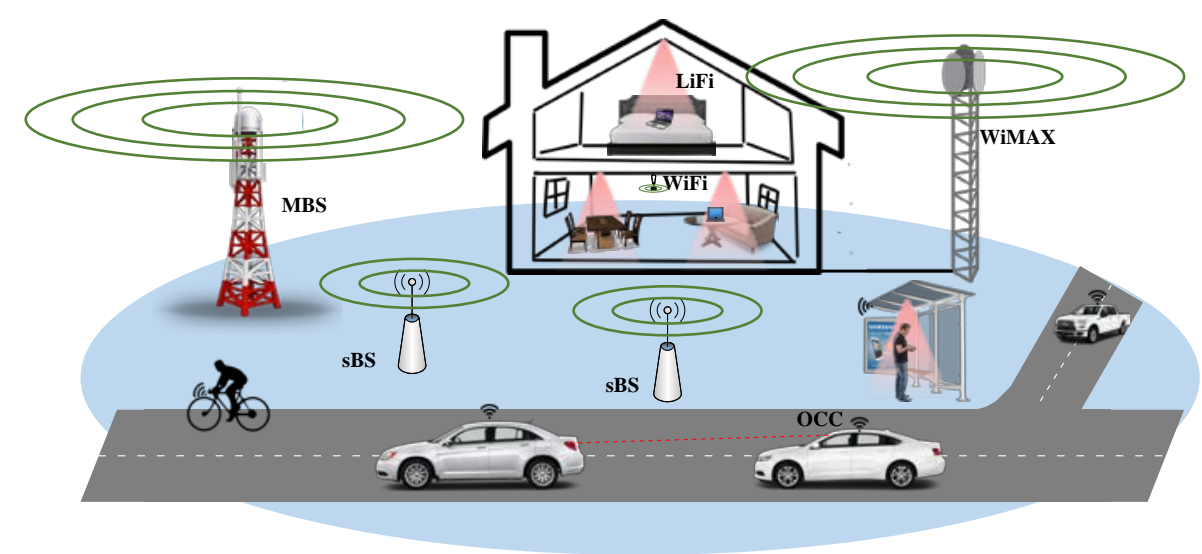

Fig. 1. Illustrative example of the network-selection problem.

\subsection{Related Works}

Access-network discovery and the selection function are discussed in [8], which is a study by third generation partnership project (3GPP) that defines the collaborative co-existence of non-3GPP and 3GPP networks. All radio-access networks (RANs) provide various services with different values of network attributes and prices. Thus, for a specific service, a user faces difficulty to select the most appropriate network. The notion of selecting the always-best-connected (ABC) network from the available access networks is discussed in [9]. 
Numerous studies on interface selection have been studied. The authors in [10] surveyed the decision criteria performed to rank existing RANs. Various researchers have presented several decision-making policies in their works using different criteria. These policies are usually based on multi-attribute decision-making (MADM), analytical hierarchy processes (AHPs), and game theory. An MADM-based technique is presented in [11]. A combination of Grey relational analysis (GRA) and AHP [12] was used to build a RAN selection algorithm in [13]. Game theory has also influenced numerous researchers to model network-selection mechanisms as games. The survey presented in [10] shows numerous features of game theory to perform network-selection problem. There are three types of games based on the interaction of the players: users-versus-users, networks-versus-users, and networks-versus-networks. The users-versus-users game is more suitable for single-RAN-resource allocation than multi-RAN-user selection. The networks-versus-networks game, in which networks compete with each other to attract users, has received much attention. The users-versus-networks game is by far the most promising for our purposes, as it considers both parties of interest. An optimal user-versus-networks game would not only ensure fulfillment of the requirements of both sides but also result in higher degrees of satisfaction.

Studies [14-16] represent a combination of game theory with AHP for best interface selection. The authors of [14] suggest a non-conflict game based on the GRA score between two WLAN networks. However, this network-versus-network approach obstructs the user's expected payoff. Studies $[15,16]$ distinguish among numerous service requests to build an efficient AHP matrix. They use bankruptcy-game-theory and evolutionary-game-theory models to perform network-selection problem. The bankruptcy-game-theory model is a type of cooperative game model. To select users among various RANs in this model, the RANs search for a coalition. Various users compete each other to select different RANs for different users in the evolutionary-game model. The authors of [17] propose an interface selection process that allows network-access probabilities to be computed according to linear-optimal control theory. Study [18] provides scheduling algorithms for mobile-video streaming with network-selection but does not consider AHP in the network-selection process. In [19], an operator-profit-maximization is formulated for efficient network-selection as well as rate control but does not consider the service requirement attributes. Study [20] provides a channel-allocation and network-selection policy but also does not consider the service requirement attributes. The authors of [21] propose a BS-selection technique for millimeter wave (mmWave) cellular networks but consider only a specific mmWave cellular network environment. In [22], a RAT-selection strategy was proposed and the Nash equilibrium (NE) of the game was formulated but the dynamic network attributes were not considered. Based on a low-complexity linear program joined with a game-theoretic approach, [23] presents a network-selection technique over HetNets. RAT-selection approach based on context-aware multi-attribute in [24] reduces the unnecessary handover. Combining AHP with game theory, these methods work for fixed weight systems but would not work well for dynamically changing weight systems.

\subsection{Contributions of This Paper}

Our proposed method considers the dynamic nature of the quality-of-experience (QoE) along with the performance requirements of various services, and so, renders the network-selection mechanism suitable for upcoming 5G network service requirements. Our proposed method takes into account the service requirements in addition to static and dynamic network attributes while using AHP to evaluate available networks. The contributions of this paper is summarized as: 
- Effective network-selection for the overlaid multi-RAN environment is presented.

- For a specific service request, a network-versus-user strategic game to perform an optimal RAN selection is provided.

- The strategic game for a particular service is formulated. Based on the static service requirements [25], to calculate the relative value of each service requirement criterion, AHP is used to form a ranking of existing various RANs. However, to determine the actual weight of different available RANs, dynamic metrics, e.g., received signal strength, obtainable bandwidth, and network load are considered.

- Each of the available RANs in its own right rates the network metrics in different ways. A cost-versus-benefit ratio is obtained to formulate the utility standards from the networks' points of view.

- The user's willingness to pay for a specific service from a specific access network considering the lowest price offered is correlated. This set is then examined to get the NEs.

- The proposed system confirms that the NE signifies the most appropriate set of solutions in terms of both the network-offered benefits and the user's willingness to pay. Thus, a user is secured in paying of high charges for the equal deal supported by different RANs with alike scores while RANs with small prices and low quality of service are disqualified.

The remainder of this paper is organized as follows. Section 2 delivers an overview of the theoretical background behind AHP and game theory. Section 3 discusses the network-selection system based on AHP and game theory. Section 4 presents illustrative examples of the evaluation of the proposed method's performance. Finally, Section 5 offers our conclusions.

\section{Overview of Theoretical Background}

\subsection{Analytic Hierarchy Process}

Developed by Thomas L. Saaty in the 1970s, AHP is a method for establishing and analyzing different complex decisions according to mathematical and psychological principles. AHP has been widely studied and applied to solve problems in different fields. A

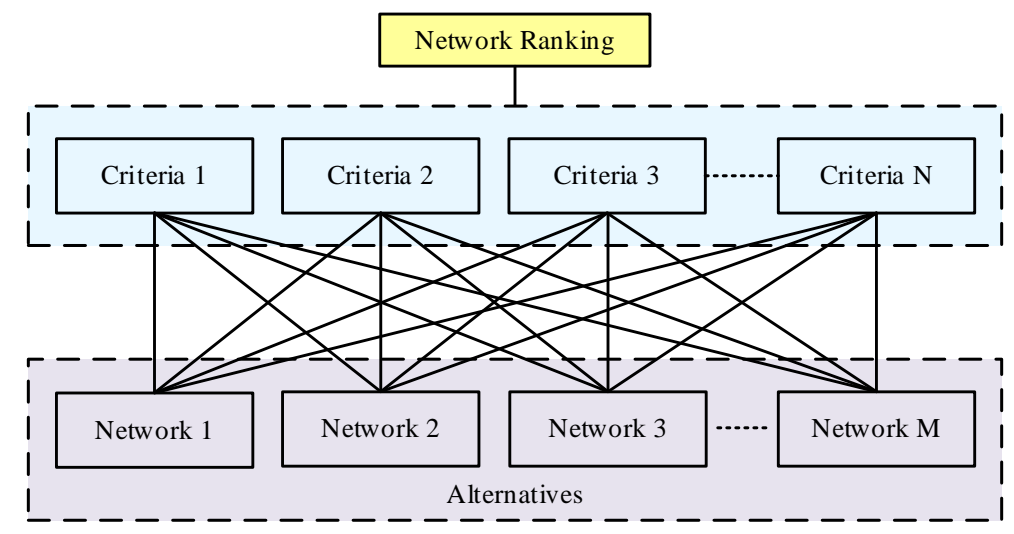

Fig. 2. Hierarchical structure of AHP for interface selection. 
Table 1. Example of relative-importance scale

\begin{tabular}{|l|c|c|c|c|c|}
\hline Importance & 1 & 3 & 5 & 7 & 9 \\
\hline Definition & Equal & Weak & Strong & $\begin{array}{c}\text { Very } \\
\text { strong }\end{array}$ & Absolute \\
\hline
\end{tabular}

multi-criteria decision-making (MCDM) method is presented in [12, 26]. MCDM process develops a hierarchy among various decision criteria and ranks the available different alternatives. The MCDM process formulates a decision matrix with several decision criteria and various alternatives. Hence, according to multiple input criteria, AHP decides the best alternative. Let us assume that, for a particular decision, the number of criteria and alternatives are $N$ and $M$, respectively. Let $a_{i j}$ denote the value of the $i^{\text {th }}$ alternative considering the $j^{\text {th }}$ criteria and $W_{j}$ be the weight of the $j^{\text {th }}$ criteria. Then, the MCDM decision-making matrix is expressed as

\begin{tabular}{|c|c|c|c|}
\hline & Criteria & & \\
\hline & $\mathrm{C}_{1} \quad \mathrm{C}_{2} \quad \mathrm{C}_{3}$ & & $\mathrm{C}_{N}$ \\
\hline Alt. & $\begin{array}{lll}W_{1} & W_{2} & W_{3}\end{array}$ & 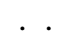 & $\mathrm{W}_{N}$ \\
\hline $\mathrm{A}_{1}$ & $a_{11} \quad a_{12} a_{13}$ & & $\mathrm{a}_{1 N}$ \\
\hline $\mathrm{A}_{2}$ & $a_{21} \quad a_{22} a_{23}$ & & $\mathrm{a}_{2 N}$ \\
\hline $\mathrm{A}_{3}$ & $a_{31} \quad a_{32} a_{33}$ & . . & $\mathrm{a}_{3 N}$ \\
\hline$\cdot$ & $\cdot \cdot \cdot \cdot$ & $\cdot \cdot$ & \\
\hline A & $\mathrm{a}_{M 1} \mathrm{a}_{M 2} \mathrm{a}_{M 3}$ & . . & $\mathrm{a}_{M N}$ \\
\hline
\end{tabular}

The relative maturity of the decision criteria is not always straightforward, thereby making the MCDM process very complex to implement. AHP presents a very powerful tool for selecting the best alternative by creating a hierarchical sub-problem approach. The goal stays at the top level of the hierarchy and the alternatives at the bottom level. In between these two levels are the criteria and sub-criteria. Fig. 2 shows the hierarchical structure of AHP. According to the $N$ criteria, the best network among the $M$ alternatives is selected.

AHP consists of multiple layers and compares all the elements of the different layers to find the best alternative. The hierarchical structure relates the elements of one level with those of the level directly below it to form relations. Then, pairwise comparisons, in which each criterion is weighed against all the other criteria and allocated computable numbers, are implemented, as shown in Table 1. The numerical values indicate the comparative rank of each criterion with respect to the others. Intermediate values, such as 2, 4, 6, and 8, can also be allocated. The next step is to construct a square matrix with values obtained from the pairwise comparisons:

$$
\left[\begin{array}{ccccc}
w_{11} & w_{12} & w_{13} & \ldots & w_{1 N} \\
w_{21} & w_{22} & w_{23} & \ldots & w_{2 N} \\
w_{31} & w_{32} & w_{33} & \ldots & w_{3 N} \\
\cdot & \cdot & \cdot & \ldots & \cdot \\
w_{N 1} & w_{N 2} & w_{N 3} & \ldots & w_{N N}
\end{array}\right]
$$

where $w$ represents the numerical values attained from individual pairwise comparison. $w_{m j}=1$ if both $m$ and $j$ criteria are of same importance or $m=j, w_{m j}>1$ if criterion $m$ is more important than $j$, and $w_{m j}<1$ if criterion $m$ is less important than $j$. 
The normalized eigenvector of the above square matrix gives the relative weights of each criterion and is written as

$$
\left[\begin{array}{c}
W_{1} \\
W_{2} \\
W_{3} \\
\cdot \\
W_{N}
\end{array}\right]
$$

AHP now performs a pairwise comparison between the alternatives with respect to a criterion objective, constructs a square matrix, and calculates the normalized eigenvectors to find the relative weights of the alternatives. The final outcome of this process is a matrix of dimensions $M \times N$ :

$$
\left[\begin{array}{cccccc}
a_{11} & a_{12} & a_{13} & \cdot & \cdot & a_{1 N} \\
a_{21} & a_{22} & a_{23} & \cdot & \cdot & a_{2 N} \\
a_{31} & a_{32} & a_{33} & \cdot & \cdot & a_{3 N} \\
\cdot & \cdot & \cdot & \cdot & \cdot & \cdot \\
a_{M 1} & a_{M 2} & a_{M 3} & \cdot & \cdot & a_{M N}
\end{array}\right]
$$

Finally, the alternatives are ranked and their global weight is found by multiplying the final two matrices:

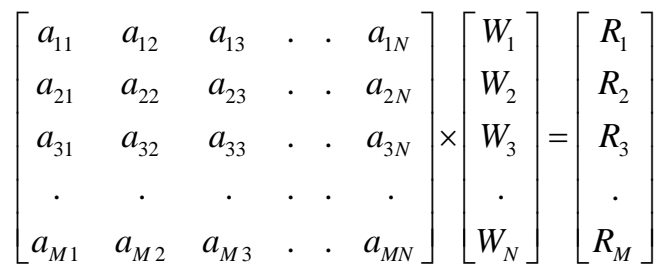

where $R$ represents the weight of the alternative.

\subsection{Strategic Game Theory}

Game theory has attracted huge interest over the past few decades in various fields of study. Although its primary use is in economics, it has become very popular in wireless-network decision-making and modeling. Among the various game models, non-cooperative game theory and the existence of equilibrium points help to establish numerous decision-making processes. Strategic game theory is a kind of non-cooperative game where each player acts rationally without any means of collaboration with other players. The outcome of individual player's choice is characterized by distinct quantitative payoff values, which are named as utilities. The worth of a utility indicates how much a player likes the related outcome. The strategic game is a collaboration among decision-makers and obeys the following three rules:

(i) A finite set of players $P_{i}=\left\{p_{1}, p_{2}, \mathrm{~L} p_{n}\right\}$;

(ii) A set of strategies or actions $S_{i}=\left\{s_{1}, s_{2}, L s_{p}\right\}$ for individual $i^{\text {th }}$ player;

(iii) The payoffs considering the set of actions $u_{i}(s)$. 
John Nash [27] proposed the solution of a non-cooperative game which is familiar as a NE. It indicates a set of strategies, $S_{i}=\left\{s_{1}, s_{2}, \mathrm{~L} s_{p}\right\}$, taken by the $i^{\text {th }}$ player to optimally respond toward the strategy of the player's opponents. Let us consider $S_{i}$ and $S_{-i}$ to be the set of strategies of the $i^{\text {th }}$ player and opponents, where $s=\left(s_{i}, s_{-i}\right) \in S$ and $u(s)=u\left(s_{i}, s_{-i}\right)$ denote the corresponding utility sets. Thus, by the definition of NE for each $i^{\text {th }}$ player and for all $s_{i}^{\prime} \neq s_{i}$

$$
u_{i}\left(s_{i}, s_{-i}\right)>u_{i}\left(s_{i}^{\prime}, s_{-i}\right)
$$

\section{Proposed Network-selection Strategy}

Efficient network-selection plays an important role in an overlaid heterogeneous network system. The best network selection or "ABC" concept has attracted the attention of various researchers toward exploring numerous features of this idea. This paper classified the network-selection strategy into two categories such as centralized and decentralized approaches.

The centralized-operator-controlled decision-making is included in the centralized [10] or the network-centric technique. Here, the network or the operator controls the policies. The subscribers are obliged to abide by the network's decision. The centralized approach largely benefits the operator; however, the user's choices and demands are somewhat neglected. The centralized approach also falls short where multiple networks of different technologies coexist at the same time. The users are registered with numerous RANs in the decentralized approach. This is in contrast to a better user-centric approach to chose the best-valued networks.

The selection of best network in a HetNets scenario is a complex decision-making procedure concerning numerous criteria as inputs of the system. Fig. 3 shows the mechanism for the best-network-selection process for the proposed scheme. It is essential to separate the decision-making various criteria into the classes of dynamic and static. The service requirement parameters are static in nature whereas the network matrices are dynamic in nature. The service requirement parameters include packet loss, delay, jitter, and bandwidth. The network matrices include dynamic nature parameters such as received signal strength power (RSSP), available bandwidth, and blocking probability. As can be seen in Fig. 3, we first consider the static application requirements of a particular service-type in our proposed scheme. AHP is used to assign a preliminary ranking to the available RANs. To develop realistic network conditions, different dynamic factors are considered. The following step comprises of finding a cost-versus-benefit ratio for the RANs. Then, a non-cooperative game is played with a cost-versus-benefit analysis and the users' readiness to pay for certain RANs for particular deal requests. The NE provides the set of service requests and the most suitable RAN. 


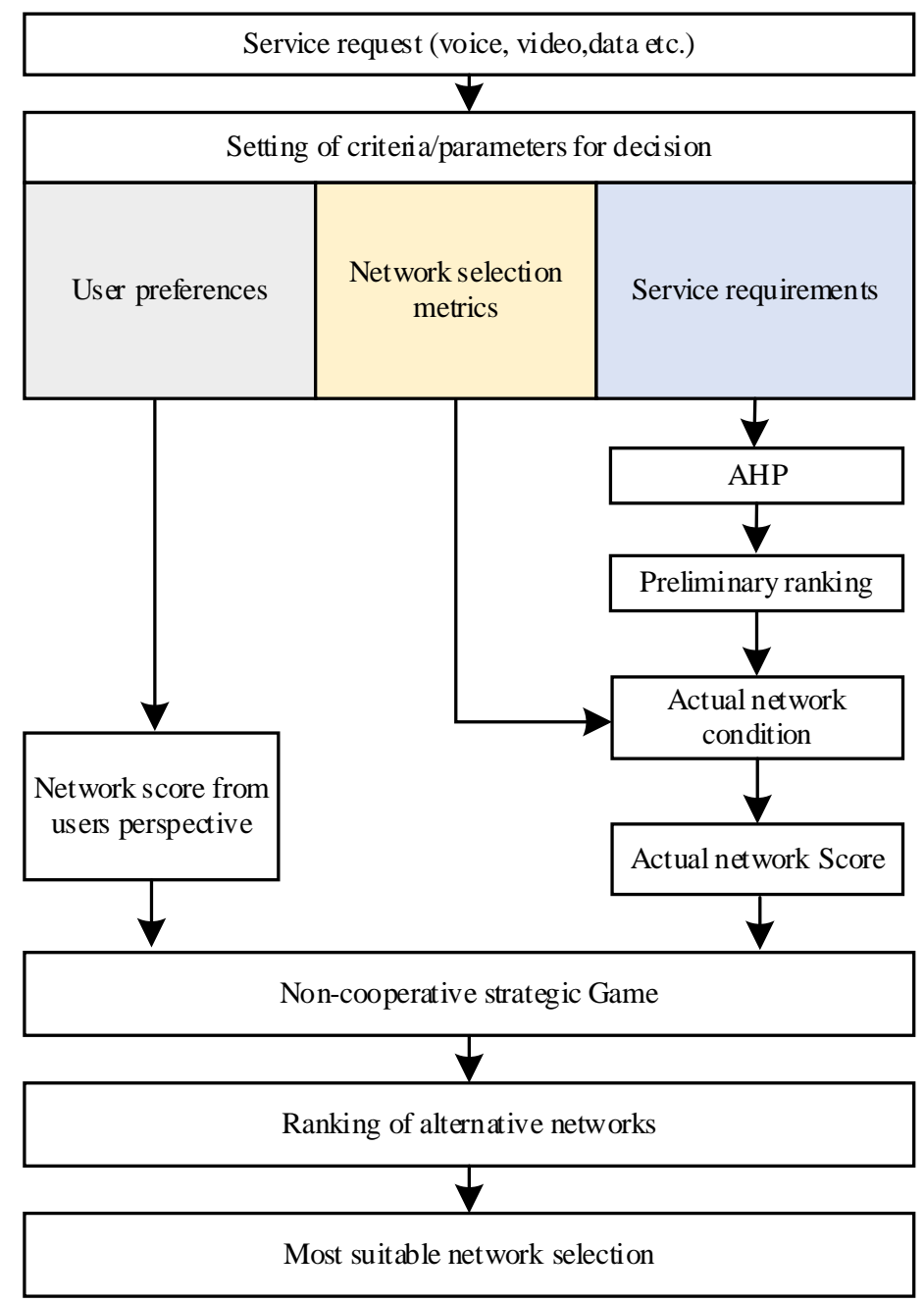

Fig. 3. Decision-making process for proposed best-network-selection strategy.

\subsection{Network Ranking}

As explained in the previous section, using AHP to establish a hierarchy among multiple alternative networks is a complex step-by-step problem involving multiple criteria, and in some cases, various sub-criteria. The pairwise comparison governing AHP requires comparative data sets to evaluate the criteria and alternatives. Firstly, it is essential to specify various criteria for diverse service requests and alternative choices of RANs for the interface selection problem. Different services require different criteria. Hence, the proper setting of the criteria is very important for achieving the best outcome. Table 2 shows a few examples of various QoE expectations and performance requirements for various types of services [1,28]. Thus, it is clear from Table 2 that, for different service types, the weights of the criteria differ. Next, we need to look at the available RANs to establish comparative weights between the criteria. Different networks provide dissimilar levels of service in terms of different criteria. 
Table 2. QoE expectations and performance requirement parameters for different service types.

\begin{tabular}{|l|l|l|}
\hline Service & QoE expectation & Key performance parameters and target values \\
\hline Conversational voice & $\begin{array}{l}\text { High (low latency } \\
\text { and low jitter) }\end{array}$ & $\begin{array}{l}\bullet \text { Bandwidth }(21-320 \mathrm{kbps} \text { per call) } \\
\bullet \text { One-way latency }<150 \mathrm{~ms} \\
\bullet \text { One-way jitter }<30 \mathrm{~ms} \\
\bullet \text { Packet loss }<3 \%\end{array}$ \\
\hline Conversational video & $\begin{array}{l}\text { High (extremely-low } \\
\text { packet loss and low } \\
\text { jitter) }\end{array}$ & $\begin{array}{l}\bullet \text { High bandwidth } \\
\bullet \text { One-way latency }<150 \mathrm{~ms} \\
\bullet \text { Packet loss }<1 \%\end{array}$ \\
\hline $\begin{array}{l}\text { Non-conversational } \\
\text { video }\end{array}$ & $\begin{array}{l}\text { High (Low jitter and } \\
\text { extremely low packet } \\
\text { loss) }\end{array}$ & $\begin{array}{l}\bullet \text { High bandwidth } \\
\bullet \text { One-way latency }<2 \text { sec } \\
\bullet \text { Packet loss }<2 \%\end{array}$ \\
\hline Web-browsing & Low (best effort) & $\begin{array}{l}\bullet \text { Consumption of variable bandwidth } \\
\bullet \text { One-way delay }<4 \text { sec /page } \\
\bullet \text { Zero loss of packet }\end{array}$ \\
\hline $\begin{array}{l}\text { Gaming and } \\
\text { interactive }\end{array}$ & $\begin{array}{l}\text { Services high (low } \\
\text { packet loss) }\end{array}$ & $\begin{array}{l}\bullet \text { Consumption of variable bandwidth } \\
\bullet \text { One-way latency }<75 \mathrm{~ms} \\
\bullet \text { Packet loss }<3 \%\end{array}$ \\
\hline Peer-to-peer & Low (best effort) & $\begin{array}{l}\bullet \text { Consumption of very high bandwidth } \\
\bullet \text { Tolerant to latency and loss }\end{array}$ \\
\hline $\begin{array}{l}\text { Enterprise/business } \\
\text { services }\end{array}$ & High (critical data) & $\begin{array}{l}\bullet \text { High bandwidth consumption } \\
\bullet \text { Highly latency sensitive } \\
\bullet \text { High security }\end{array}$ \\
\hline $\begin{array}{l}\text { Transaction services } \\
\text { e.g. e-commerce, } \\
\text { ATM }\end{array}$ & High (critical data) & $\begin{array}{l}\text { •One-way latency }<4 \text { sec } \\
\bullet \text { Zero packet loss }\end{array}$ \\
\hline E-mail & Low (best effort) & $\begin{array}{l}\bullet \text { One-way latency }<4 \text { sec } \\
\bullet \text { Zero packet loss }\end{array}$ \\
\hline
\end{tabular}

Table 3. Network attributes for various available alternative RANs [25].

\begin{tabular}{|c|c|c|c|c|c|}
\hline Network & PD (ms) & PJ (ms) & $\begin{array}{c}\text { PL } \\
(\text { per 10 })\end{array}$ & S (\%) & BR (Mbps) \\
\hline RAN1 & $25-50$ & $5-10$ & $20-80$ & 60 & $0.1-2.0$ \\
\hline RAN2 & $60-100$ & $3-10$ & $20-80$ & 60 & $1.0-60.0$ \\
\hline RAN3 & $100-150$ & $10-20$ & $20-80$ & 40 & $1.0-11.0$ \\
\hline RAN4 & $60-100$ & $3-10$ & $20-80$ & 50 & $1.0-60.0$ \\
\hline
\end{tabular}

Table 3 shows an example of network attributes such as packet delay (PD), packet loss (PL), packet jitter (PJ), security (S), and bit rate (BR) for four different RANs. AHP is used to rank the offered various RANs considering different criteria for a service request. The criterion assessment is accomplished considering the QoE prospects and performance requirements listed in Table 2. The pairwise comparison and assignment of weights according to Table 1 help in the building of the matrix in (1). The eigenvectors of this matrix in (2) give weights to the criteria for the indicated service-type. The subsequent step is to compare the alternative various RANs considering the criteria and construct the matrix as in (3). The final weight of the alternative RANs is calculated by using (4) to create a preliminary ranking of the available networks. 


\subsection{Actual Network Score Calculation}

For a specific service request, the weight estimation of a RAN considers the static parameters. It is required to combine the asessment for the dynamic parameters of various RANs with the computed AHP score by considering the static parameters to weigh existing RANs. Therefore, for a candidate RAN, the actual weight is expressed as

$$
W_{\text {RAN }}=\sum \alpha \cdot R_{A H P}
$$

where $\alpha \in w_{X}, X \in\{R S S P$, avilable BW, call blocking probability $\}$, and $w_{X}=\frac{\text { Actual value }}{\text { Best value }}$.

\subsection{Non-cooperative Strategic Game Formation}

Up to this point, what we have is a hierarchy of the available RANs considering both the static as well as dynamic attributes of requested services and available networks. However, the user's budget and/or the willingness to pay for a specific service demand is also another factor that impacts the selection of RANs. The selection of the optimal RAN not only needs to fulfill the best service conditions but also needs to consider the economic aspect. For a user point of view, the choice of the best RAN at the best probable price is the most optimal output. Whatever lower than this outcome may cause to a Pareto-inefficient outcome. As a result of Pareto-inefficient outcome, a user receives less benefit against paying of same value. Otherwise, the user may be encouraged to pay higher prices to receive the same benefit. Therefore, for the RAN selection, a two-player non-cooperative strategic game containing the rules is proposed as follows:

(i) We consider one player for network plane and another payer for user plane. To choice the best network, these players participate in the game without any form of collaboration.

(ii) The strategy set $S_{i}$ is not same for diverse players. It is the accessible alternative RANs for the network plane, whereas it is the user's requested services for the user plane. Thus, $S_{\text {Network plane }} \in\left\{R A N_{1}, R A N_{2}, \mathrm{~L} R A N_{M}\right\}$ and $S_{\text {user plane }}$ are the sets of requested services.

(iii)The payoffs for individual set of actions or strategies for diverse players also differ. For the network plane, the payoff is the cost-benefit ratio obtainable for a specific requested service. For the user plane, it is the user's willingness to pay for the requested service of a specific network.

Let consider player $P_{1} \in$ Network and $P_{2} \in$ user, as well as their available strategy sets, be $S_{1} \in\left\{R A N_{1}, R A N_{2}, R A N_{3}, \mathrm{~L} R A N_{M}\right\}$ and $S_{2} \in \operatorname{service}$ requests $\left(r_{1}, r_{2}, r_{3}, \mathrm{~L}, r_{N}\right)$., respectively. The payoff functions for different players are also different. Let consider the payoff function for the players be $u_{1}\left(S_{1}\right)$ and $u_{2}\left(S_{2}\right)$, respectively. We use the actual weights of the RANs and the prices they charge for a specific service request. The payoff function of player $P_{1}$ can be written as:

$$
u_{R A N_{m}, r_{n}}=\frac{W_{R^{\prime} N_{m}, r_{q}}}{\bar{C}_{R A N_{m}, r_{q}}},
$$

where $R A N_{m}$ and $r_{q}$ indicate that a service request $r_{q}$ is served by $R A N_{m}$ and its weight is $W$, as in (6). $\bar{C}_{R A N_{m}, r_{q}}$ is the normalized cost for the service request $r_{q}$ which is served through $R A N_{m}$. 


\begin{tabular}{|c|c|c|c|c|c|c|c|}
\hline & & \multicolumn{6}{|c|}{ User Plane } \\
\hline & & $r_{1}$ & $r_{2}$ & $r_{3}$ & $r_{4}$ & $\ldots$ & $r_{N}$ \\
\hline \multirow{5}{*}{ 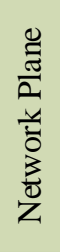 } & $R A N_{1}$ & $u_{R A N, 1, r 1}$ & $u_{R A N, 1, r 2}$ & $u_{R A N, 1, r 3}$ & $u_{R A N, 1, r 4}$ & $\ldots$ & $u_{R A N, 1, r N}$ \\
\hline & $\vdots$ & $\vdots$ & $\vdots$ & $\vdots$ & $\vdots$ & $\ldots$ & . \\
\hline & $R A N_{q}$ & $u_{R A N, q, r 1}$ & $u_{R A N, q, r 2}$ & $u_{R A N, q, r 3}$ & $u_{R A N, q, r 4}$ & $\ldots$ & $u_{R A N, q, r N}$ \\
\hline & $\vdots$ & $\vdots$ & $\vdots$ & $\vdots$ & $\vdots$ & $\ldots$ & . \\
\hline & $R A N_{M}$ & $u_{R A N, M, r 1}$ & $u_{R A N, M, r 2}$ & $u_{R A N, M, r 3}$ & $u_{R A N, M, r 4}$ & $\ldots$ & $u_{R A N, M, r N}$ \\
\hline
\end{tabular}

Fig. 4. Game space for users for a network game with payoff.

Thus $\bar{C}_{R A N_{m}, r_{q}}=\frac{C_{R_{a n}, r_{q}}}{\sum_{m=1}^{M} C_{R A N_{m}, r_{q}}}$, where $C_{R A N_{p}, r_{q}}$ is the actual cost charged by the RAN and $\sum_{m=1}^{M} C_{R A N_{m}, r_{q}}$ is the sum of the distinct costs for all $M$ RANs accessible to offer service $r_{q} . P_{2}$ is the willingness to pay for a specifc service under a specific RAN. The score of an absolute willingness is 1 . It specifies that the user's evaluation of a service considering the cost and the service provider's actual price are the same. Therefore, the willingness to pay (WTP) can be expressed as

$$
W T P=1+\frac{P_{u}-P_{a}}{P_{u}}
$$

where $P_{u}$ and $P_{a}$ are the user's perceived price and the actual price, respectively.

The equilibrium strategies that set off the game-satisfying condition in (5) are the NEs of this game and signify the most appropriate RAN for a specific requested service. The game space takes the system presented in Fig. 4. The outcome of the game is the choice of the NEs originated in each column. An NE is a strategy with the maximum payoff in an offered set. Therefore, in this game, a strategy causing in an NE contains a service request $r_{q}$ along with its most suitable network $R A N_{p}$.

Even though this paper considers the service requirements, both the static as well as dynamic network attributes, and the combination of AHP with the game theory, the processing time for the proposed scheme will not suffice to achieve the required goal. The processing time depends on the system configurations. The currently available mobile devices, as well as network systems, are fast enough to support the short processing time of the proposed interface selection method.

\section{Illustrative Examples}

This section provides an illustrative example of the network-selection approach proposed in this paper. The simulation environments are numeric iterations based on the network QoE expectations, the requirements of various service types (refer to Table 2), and network attributes for various available RANs (refer to Table 3). The criteria-weight matrices for service-type 1, service-type 2, and service-type 3, as well as the steps presented in Sections 2 and 3 for the most suitable network-selection, are described in this section.

Three different services, namely service 1 , service 2 , and service 3 , are considered for this illustration. The performance requirements for the three different types of service requests are 
shown in Table 2. It is assumed that a user demanding these services is listed into the multi-technology heterogeneous networks containing RAN1, RAN2, RAN3, and RAN4. Our provided scheme supports the universal approach in RAN selection. Therefore, without concentrating on specific RANs, such as long-term evolution (LTE) and WiMAX, the proposed system provides a more general approach by considering the RANs. For the illustrative examples, we assume that four different RANs are available to serve for a particular service request. RAN1 and RAN2 are modeled on LTE standard, RAN3 is considered as WLAN standard, and RAN4 is based on the WiMAX standard. The corresponding network attributes for different RANs are listed in Table 3.

PD, PJ, PL, S, and BR are the assumed static attributes. The primary mission is to follow different steps of Sections 2 and 3 to accomplish an AHP investigation. For service-type 1, the criteria-weight matrix is

$$
\begin{array}{cccccc} 
& P D & P J & P L & B R & S \\
P D & 1 & 5 / 1 & 7 / 1 & 5 / 1 & 7 / 1 \\
P J & 1 / 5 & 1 & 3 / 1 & 7 / 1 & 5 / 1 \\
P L & 1 / 7 & 1 / 3 & 1 & 1 / 3 & 3 / 1 \\
B R & 1 / 5 & 1 / 7 & 3 / 1 & 1 & 1 / 2 \\
S & 1 / 7 & 1 / 5 & 1 / 3 & 2 / 1 & 1
\end{array}
$$

For service-type 2, this matrix is

$$
\begin{array}{cccccc} 
& P D & P J & P L & B R & S \\
P D & 1 & 1 / 5 & 1 / 7 & 1 / 5 & 2 / 1 \\
P J & 5 / 1 & 1 & 1 / 3 & 3 / 1 & 5 / 1 \\
P L & 7 / 1 & 3 / 1 & 1 & 7 / 1 & 9 / 1 \\
B R & 5 / 1 & 1 / 3 & 1 / 7 & 1 & 1 / 2 \\
S & 1 / 2 & 1 / 5 & 1 / 9 & 2 / 1 & 1
\end{array}
$$

For service-type 3, it is

$$
\begin{array}{cccccc} 
& P D & P J & P L & B R & S \\
P D & 1 & 1 / 3 & 1 / 7 & 1 / 3 & 1 \\
P J & 3 / 1 & 1 & 1 / 3 & 5 / 1 & 5 / 1 \\
P L & 7 / 1 & 3 / 1 & 1 & 5 / 1 & 7 / 1 \\
B R & 3 / 1 & 1 / 5 & 1 / 5 & 1 & 1 / 3 \\
S & 1 & 1 / 5 & 1 / 7 & 3 / 1 & 1
\end{array}
$$

From the normalized eigenvectors of these achieved three matrices, the criteria weights for the three service types are obtained, as in (2). Table 4 summarizes the criteria weights for individual type of service.

Table 4. Criteria weights for various service types

\begin{tabular}{|c|c|c|c|}
\hline Criteria & Service 1 & Service 2 & Service 3 \\
\hline PD & 0.5312 & 0.0605 & 0.0612 \\
\hline PJ & 0.2475 & 0.2398 & 0.2655 \\
\hline PL & 0.0762 & 0.5251 & 0.4990 \\
\hline BR & 0.0813 & 0.1033 & 0.0808 \\
\hline S & 0.0635 & 0.0711 & 0.0930 \\
\hline
\end{tabular}


The subsequent step is to accomplish pairwise comparisons between the existing RANs based on different criteria. The matrix formed by considering the PD, PD, PL, BR, and S criteria is created based on available data in Table 3.

For the criterion PD, the square matrix for the available RANs is

$\begin{array}{ccccc}P D & R A N_{1} & R A N_{2} & R A N_{3} & R A N_{4} \\ R A N_{1} & 1 & 1 & 7 / 1 & 5 / 1 \\ R A N_{2} & 1 & 1 & 7 / 1 & 5 / 1 \\ R A N_{3} & 1 / 7 & 1 / 7 & 1 & 1 / 3 \\ R A N_{4} & 1 / 5 & 1 / 5 & 3 / 1 & 1\end{array}$

For the criterion PJ, the square matrix for the available RANs is

$\begin{array}{ccccc}P J & R A N_{1} & R A N_{2} & R A N_{3} & R A N_{4} \\ R A N_{1} & 1 & 1 & 6 / 1 & 1 / 2 \\ R A N_{2} & 1 & 1 & 6 / 1 & 1 / 2 \\ R A N_{3} & 1 / 6 & 1 / 6 & 1 & 1 / 7 \\ R A N_{4} & 2 / 1 & 2 / 1 & 7 / 1 & 1\end{array}$

For the criterion PL, the square matrix for the available RANs is

$\begin{array}{ccccc}P L & R A N_{1} & R A N_{2} & R A N_{3} & R A N_{4} \\ R A N_{1} & 1 & 1 & 1 & 1 \\ R A N_{2} & 1 & 1 & 1 & 1 \\ R A N_{3} & 1 & 1 & 1 & 1 \\ R A N_{4} & 1 & 1 & 1 & 1\end{array}$

For the criterion BR, the square matrix for the available RANs is

$\begin{array}{ccccc}B R & R A N_{1} & R A N_{2} & R A N_{3} & R A N_{4} \\ R A N_{1} & 1 & 1 & 1 / 7 & 1 / 9 \\ R A N_{2} & 1 & 1 & 1 / 7 & 1 / 9 \\ R A N_{3} & 7 / 1 & 7 / 1 & 1 & 1 / 7 \\ R A N_{4} & 9 / 1 & 9 / 1 & 7 / 1 & 1\end{array}$

For the criteria $\mathrm{S}$, the square matrix for the available RANs is

$\begin{array}{ccccc}S & R A N_{1} & R A N_{2} & R A N_{3} & R A N_{4} \\ R A N_{1} & 1 & 1 & 5 / 1 & 3 / 1 \\ R A N_{2} & 1 & 1 & 5 / 1 & 3 / 1 \\ R A N_{3} & 1 / 5 & 1 / 5 & 1 & 1 / 3 \\ R A N_{4} & 1 / 3 & 1 / 3 & 3 / 1 & 1\end{array}$

The relative weights of different accessible RANs considering different criteria are calculated from the matrix as in (3):

$\begin{array}{cccccc} & P D & P J & P L & B R & S \\ R A N_{1} & 0.4224 & 0.2529 & 1 & 0.0458 & 0.3898 \\ R A N_{2} & 0.4224 & 0.2529 & 1 & 0.0458 & 0.3898 \\ R A N_{3} & 0.0506 & 0.0487 & 1 & 0.2187 & 0.0679 \\ R A N_{4} & 0.1044 & 0.4454 & 1 & 0.6894 & 0.1523\end{array}$




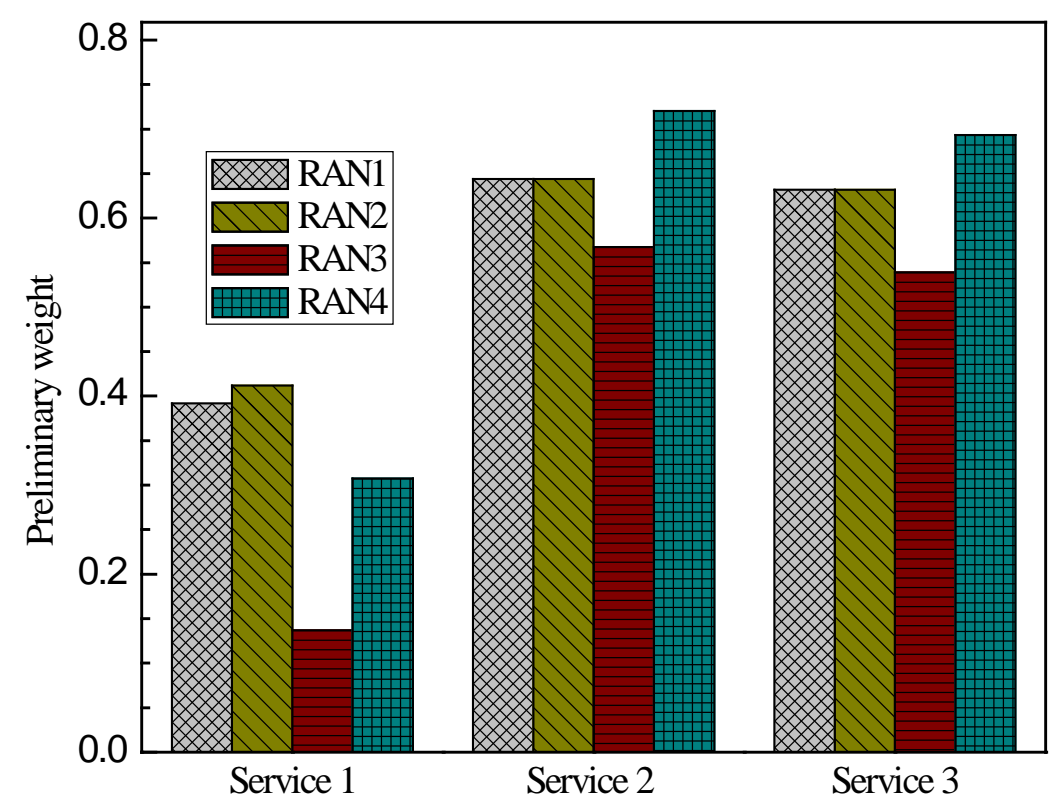

Fig. 5. Comparison of preliminary weights based on static parameters.

Fig. 5 shows the graphical illustration of preliminary weights based on static parameters for different services on different networks. As shown in Fig. 5, if the RAN selection is performed based only on the static parameters, the best-valued networks for service-type 1 are RAN1 and RAN2. RAN4 is the preeminent selection for both service types 2 and 3. Nevertheless RAN3 is the worst provider for service 1, it increases ground for service types 2 and 3 because of its higher average BR. The weight of the available RANs presented in Fig. 5 only considers the static requirements for different service requests and compares the available RANs with respect to those attributes. As mentioned before, the combination of both the static and dynamic attributes is considered for the actual network score calculation in our proposed system.

The total payoff under different conditions is calculated. The total payoff for service type 1 generated with four available RANs is presented in Fig. 6. For the same weights of RSSP and WTP by the user, the equilibrium points are generated by RAN1 and RAN2. Therefore, for service-type 1, a user chooses either RAN1 or RAN2 in this case. The choice between RAN1 and RAN2 is subjected to the offered RSSP weight under this condition. Furthermore, we observe that, the payoff generated by RAN2 far outperforms that of RAN1 with WTP $=0.7$ and $\mathrm{WTP}=0.8$ for RAN1 and RAN2, respectively. This performance shows that the NE of the game lies with RAN2. RAN4 provides a weight that is slightly close to those of RAN1 and RAN2. However, for the same RSSP values, the user is only attracted if WTP $=0.85$, i.e., there is an $18 \%$ lower price for the equal level of service 1 . Due to the worst-network score's drifting toward the most-favorable price for the user, RAN3 fails to attract any user when other RANs are available. These mechanisms prove the usefulness of our proposed system in protecting the user from choosing a RAN as a result of considering only lower price without considering the weight of network. 


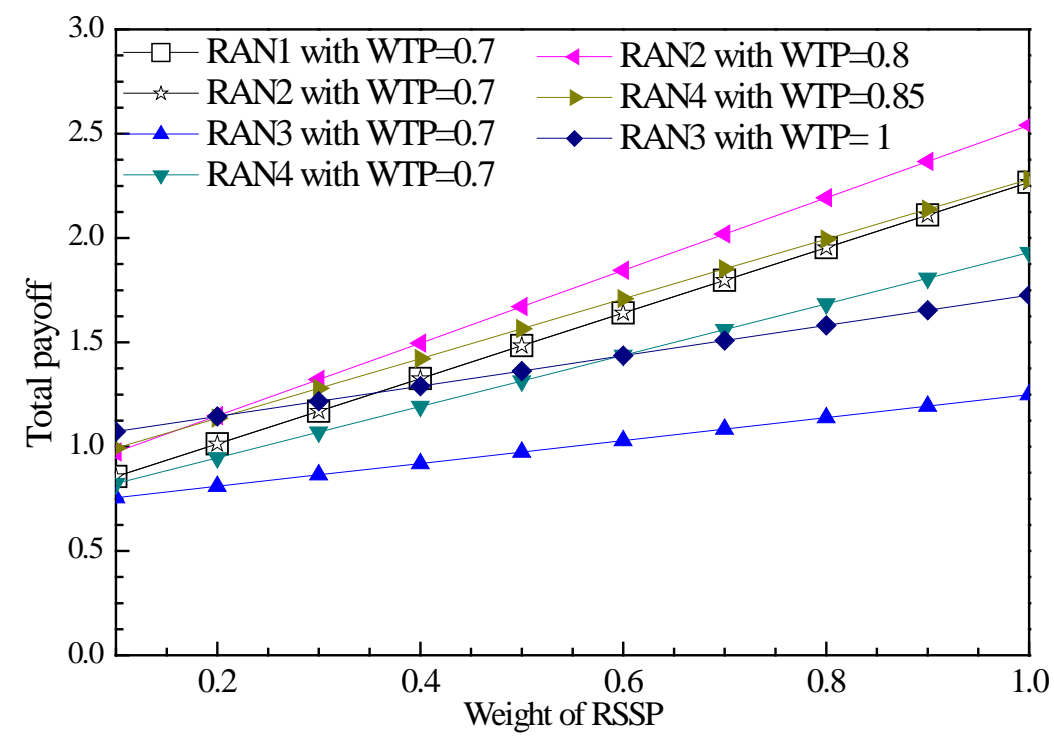

Fig. 6. Payoffs generated by strategic game for different values of RSSP weight and WTP for service-type 1.

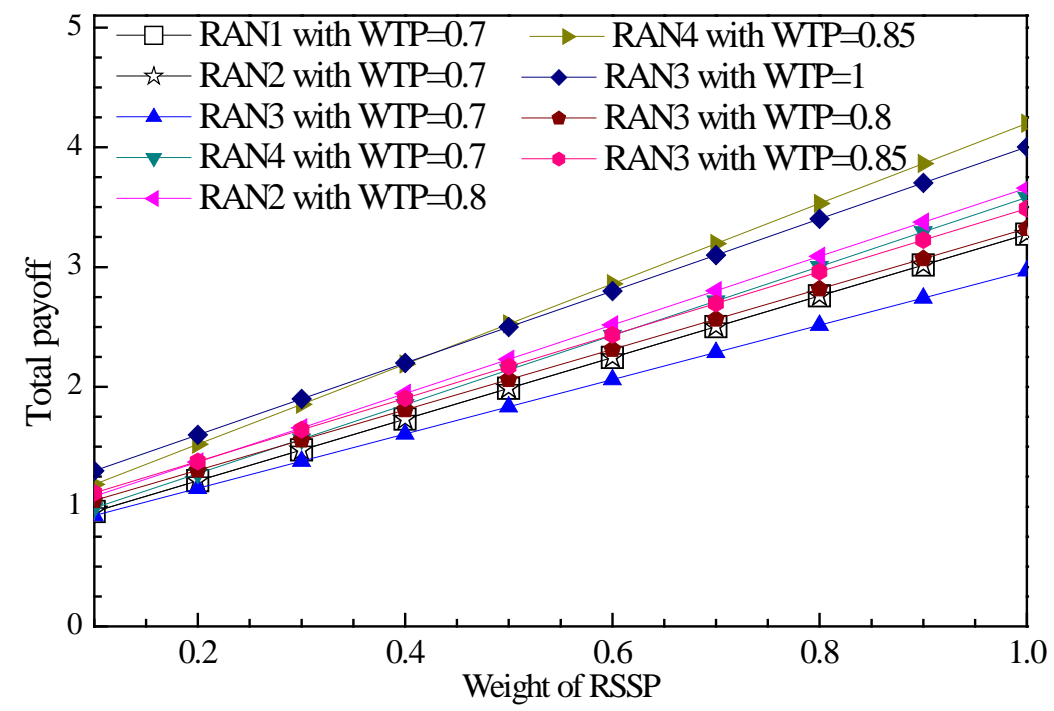

Fig. 7. Payoffs generated by strategic game for different values of RSSP weight and WTP for service-type 2.

The preliminary network weights of the RANs for service-type 2 makes RAN4 more suitable for this type of service. However, if we look at the other network scores, we can see that they are not at a very bad level. The network-selection game effectively dominates the decision-making in this scenario. Fig. 7 shows the total payoff generated by this game for service-type 2. For example, with the same RSSP weight and WTP, RAN4 is the best-suited network; however, RAN1 and RAN2 gain ground when the RSSP offered by RAN4 changes for the worst. We can also see that, with $\mathrm{WTP}=0.8$, the RAN3 payoff matches the payoffs generated with WTP=0.7 for both RAN1 and RAN2. 


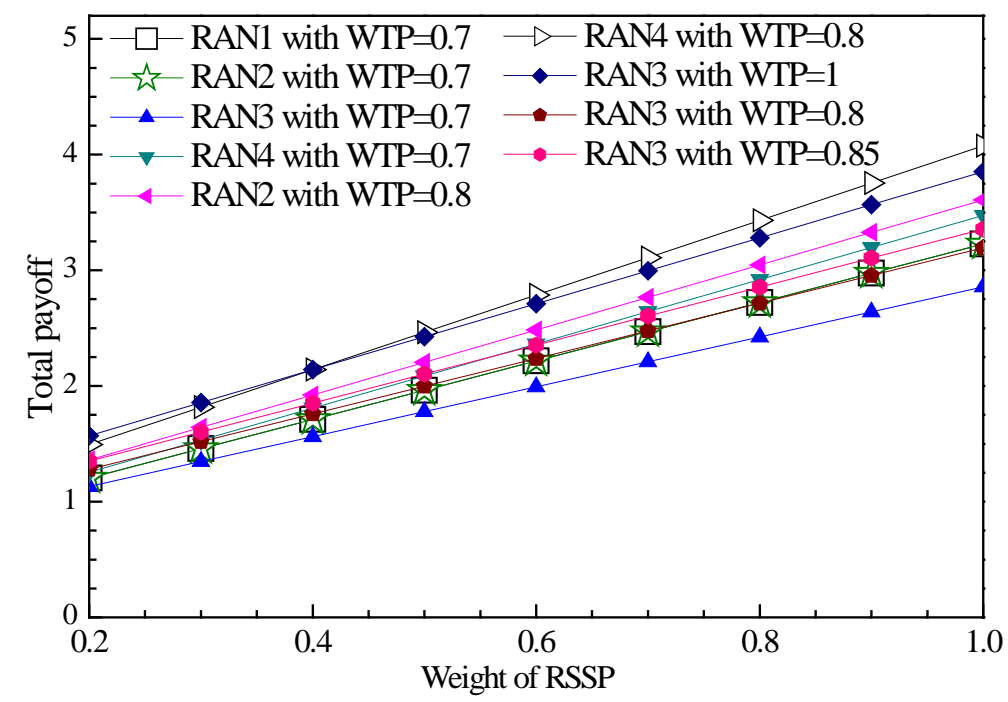

Fig. 8. Payoffs generated by strategic game for different values of RSSP weight and WTP for service-type 3.

A dynamic condition in which the payoffs for various RANs considering RSSP and WTP values for service-type 3 is presented in Fig. 8. The payoffs for different networks are: 2.72 for RAN1 with RSSP weight $=0.8$ and WTP $=0.7 ; 2.91$ for RAN4 with RSSP weight $=0.8$ and WTP $=0.7 ; 3.04$ for RAN2 with RSSP weight $=0.8$ and WTP $=0.8$; 2.85 for RAN3 with RSSP weight $=0.8$ and WTP $=0.85$. Therefore, for various RSSP and WTP weights, the payoffs indicate changes in NE, thereby providing the user with a strategic advantage over the networks. The user's decision changes along with the static or dynamic attributes of the different RANs, which need to adjust their prices in order to retain their users. This game confirms that the user receives assured level of services.

We present our results for the various combinations of WTP values in observing how our proposed scheme works. We kept WTP=0.7 for RAN1, RAN2, RAN3, and RAN4 in all cases. The WTP values for RAN2, RAN3, and RAN4 are changed with different combinations for a comparison of these results with the results of WTP=0.7 for RAN1, RAN2, RAN3, and RAN4. Figs. 6-8 show that our proposed method works well for all combinations. The proposed scheme combines the hierarchical evaluations of the networks with game theory to solve the RAN selection issue by considering the dynamic nature of the QoE expectations and performance requirements for different services. As depicted in Fig. 3, our proposed method takes into account the service requirements, as well as static and dynamic network attributes, while using AHP to evaluate the available networks. From the detailed illustrations provided in Figs. 6-8 show that the network-selection approach in this paper provides the user the upper hand for selecting the best RAN. Though it has been designed for the selection of the best-valued network, the method confirms the commitments of the RANs to their users, who receive the best service for the lowest price.

\section{Conclusions}

To achieve the goals of the convergence of HetNets, best-network-selection according to service criteria is one of the most vital issues. Hence, the issue of the best-valued interface selection requires more attention than ever, due to tha fact that the wireless technologies are 
getting more diverse for 5G-and-beyond communications. The existing network-selection methods combine AHP with game theory and consider fixed weight systems for ranking the available networks. Our proposed scheme not only combines AHP and game theory but also considers the dynamic manner of QoE expectations and service requirements. The inclusion of these additional parameters for network selection does not affect the delay. Our scheme provides parallel processing of these parameters with other procedures. We consider static and dynamic network attributes, as well as service requirements, while using AHP to evaluate the available networks. Then, we formulate a user-versus-network game to select the best suitable network.

This work distinguishes between various service types as well as their requirements during best network-selection. The proposed scheme assigns weights to the available various RANs by considering both the static and dynamic types of parameters. The proposed approach confirms that the RANs are weighted not only according to their technological attributes but also their actual radio conditions. The illustrative examples show that the network-selection approach offered in this paper gives the user the upper hand when selecting networks. Though it is designed for the selection of the best-valued network, this work ensures the commitment of a RAN to its users, i.e., ensuring that the RAN keeps its promises to its users and avoids the risk of its users drifting toward other RANs. Therefore, this system not only offers an effective way to select from different RANs but also persuades the RANs to keep their quality high along with low price. This work provides a generalized approach to network-selection. For 5G-and-beyond communication systems, this proposed dynamic network-selection approach will perform well beyond the reach of the traditional methods of network-selection. Therefore, we expect that our work would significantly contribute to the future deployment of high-density HetNets.

\section{Conflicts of Interest}

The authors declare that they have no conflicts of interest.

\section{References}

[1] Cisco visual networking index: global mobile data traffic forecast Update. (2018) [Online]. Available Online:

https://www.cisco.com/c/en/us/solutions/collateral/service-provider/visual-networkingindex-vni/mobile-white-paper-c11-520862.html

[2] M. Z. Chowdhury, Y. M. Jang, and Z. J. Haas, "Cost-effective frequency planning for capacity enhancement of femtocellular networks," Wireless Personal Communications, vol. 60, no. 1, pp. 83-104, Sept. 2011. Article (CrossRef Link).

[3] M. Z. Chowdhury and Y. M. Jang, "Handover management in high-dense femtocellular networks," EURASIP Journal on Wireless Communications and Networking, pp. 1-21, Jan. 2013. Article (CrossRef Link).

[4] Z. Ghassemlooy, S. Arnon, M. Uysal, Z. Xu, and J. Cheng, "Emerging optical wireless communications-advances and challenges," IEEE Journal on Selected Areas in Communications, vol. 33, no. 9, pp. 1738-1749, Sep. 2015. Article (CrossRef Link).

[5] I. Takai, T. Harada, M. Andoh, K. Yasutomi, K. Kagawa, and S. Kawahito, "Optical vehicle-to-vehicle communication system using LED transmitter and camera receiver," IEEE Photonics Journal, vol. 6, no. 5, pp. 1-14, Oct. 2014. Article (CrossRef Link). 
[6] M. Z. Chowdhury, M. S. Uddin, and Y. M. Jang, "Dynamic channel allocation for class-based QoS provisioning and call admission in visible light communication,” The Arabian Journal for Science and Engineering, vol. 39, no. 2, pp. 1007-1016, Feb. 2014. Article (CrossRef Link).

[7] M. Z. Chowdhury, M. T. Hossan, A. Islam, and Y. M. Jang, "A comparative survey of optical wireless technologies: architectures and applications,” IEEE Access, vol. pp. no 99, pp.1-1, 2018. https://doi.org /10.1109/ACCESS.2018.2792419

[8] 3GPP TS 24.312 v10.3.0, Access network discovery and selection function (ANDFS) management object (MO), 2011.

[9] E. Gustafsson, and A. Jonsson, “Always best connected,” IEEE Wireless Communications, vol. 10, no. 1, pp. 49-55, Feb. 2003. Article (CrossRef Link).

[10] R. Trestian, O. Ormond, and G. M. Muntean, "Game theory - based network selection: solutions and challenges,” IEEE Communications Survey \& Tutorials, vol. 14, no. 4, pp. 1212-1231, Oct. 2012. Article (CrossRef Link).

[11] L. Wang, and D. Binet, "MADM-based network selection in heterogeneous wireless networks: a simulation study," in Proc. of IEEE Wireless Communication, Vehicular Technology, Information Theory and Aerospace \& Electronics Systems Technology, pp. 559-564, May 2009. Article (CrossRef Link).

[12] E. Huang, S. Zhang, L. H. Lee, E. P. Chew, and C. H. Chen, "Improving analytic hierarchy process expert allocation using optimal computing budget allocation," IEEE Transactions on Systems, Man, and Cybernetics: Systems, vol. 46, no. 8, pp. 1140-1147, Aug. 2016. Article (CrossRef Link).

[13] Q. Song, and A. Jamalipour, "Network selection in an integrated wireless LAN and UMTS environment using mathematical modelling and computing techniques," IEEE Wireless Communications, vol. 12, no. 3, pp. 42-48, June 2005. Article (CrossRef Link).

[14] D. Charilas, O. Markaki, and E. Tragos, "A theoretical scheme for applying game theory and network selection mechanisms in access admission control," in Proc. of IEEE International Symposium on Wireless Pervasive Computing, pp. 303-307, May 2008. Article (CrossRef Link).

[15] B. Liu, H. Tian, B. Wang, and B. Fan, "AHP and game theory based approach for network selection in heterogeneous wireless networks," in Proc. of IEEE Consumer Communications and Networking Conference (CCNC), pp. 501-506, Jan. 2014. Article (CrossRef Link).

[16] N. P. Singh, "Efficient network selection using game theory in a heterogeneous wireless network," in Proc. of IEEE International Conference on Computational Intelligence and Computing Research (ICCIC), pp. 1-4, Dec. 2015. Article (CrossRef Link).

[17] Y. Li, Y. Hadjadj-Aoul, P. Bertin, and G. Rubino, "A control theoretic strategy for intelligent interface selection in heterogeneous network environments," in Proc. of IEEE Annual Consumer Communications \& Networking Conference (CCNC), pp. 1006-1011, Jan. 2016. Article (CrossRef Link).

[18] J. Lee, K. Lee, C. Han, T. Kim, and S. Chong, "Resource-efficient mobile multimedia streaming with adaptive network selection,” IEEE Transactions on Multimedia, vol. 18, no. 12, pp. 2517-2527, Dec. 2016. Article (CrossRef Link).

[19] Y. Li, B. Shen, J. Zhang, X. Gan, J. Wang, and X. Wang, “Offloading in HCNs: congestion-aware network selection and user incentive design,” IEEE Transactions on Wireless Communications, vol. 16, no. 10, pp. 6479-6492, Oct. 2017. Article (CrossRef Link).

[20] N. Ul Hasan, W. Ejaz, N. Ejaz, H. S. Kim, A. Anpalagan, and M. Jo, "Network selection and channel allocation for spectrum sharing in 5G heterogeneous networks," IEEE Access, vol. 4, pp. 980-992, 2016. Article (CrossRef Link).

[21] C. Skouroumounis, C. Psomas, and I. Krikidis, "Low-complexity base station selection scheme in mmWave cellular networks," IEEE Transactions on Communications, vol. 65, no. 9, pp. 4049-4064, Sept. 2017. Article (CrossRef Link).

[22] P. Naghavi, S. Hamed Rastegar, V. Shah-Mansouri, and H. Kebriaei, "Learning RAT selection game in 5G heterogeneous networks," IEEE Wireless Communications Letters, vol. 5, no. 1, pp. 52-55, Feb. 2016. Article (CrossRef Link). 
[23] A. Awad, A. Mohamed, and C. F. Chiasserini, "Dynamic network selection in heterogeneous wireless networks: a user-centric scheme for improved delivery," IEEE Consumer Electronics Magazine, vol. 6, no. 1, pp. 53-60, Jan. 2017. Article (CrossRef Link).

[24] A. Habbal, S. I. Goudar, and S. Hassan, "Context-aware radio access technology selection in 5G ultra dense networks,” IEEE Access, vol. 5, pp. 6636-6648, 2017. Article (CrossRef Link).

[25] M. T. Rahman, M. Z. Chowdhury, and Y. M. Jang, "Radio access network selection mechanism based on hierarchical modelling and game theory," in Proc. of International Conference on Information and Communication Technology Convergence (ICTC), pp. 126-131, Oct. 2016. Article (CrossRef Link).

[26] R. W. Saaty, "The analytic hierarchy process- what it is and how it is used," Mathematical Modelling, vol. 9, no. 3, pp. 161-176, 1987. Article (CrossRef Link).

[27] J. Nash, "Non-Cooperative Games," The annals of mathematics, vol. 54, no. 2, pp. 286-295, Sept. 1951. Article (CrossRef Link).

[28] 3GPP TS 22.105 V14.0.0, Services and service capabilities, March 2017.

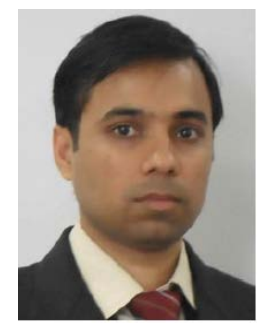

Mostafa Zaman Chowdhury obtained his B.Sc. degree in Electrical and Electronic Engineering from Khulna University of Engineering and Technology (KUET), Bangladesh, in 2002. He obtained his M.Sc. and Ph.D. degrees both in Electronics Engineering from Kookmin University, Korea, in 2008 and 2012, respectively. In 2003, he joined the Electrical and Electronic Engineering department at KUET as a Lecturer where he is currently affiliated as Professor. He has been working as a postdoc researcher at Kookmin University, Korea since 2017. He is a senior member of IEEE. In 2008, he received the Excellent Student Award from Kookmin University. He has published around 125 research papers in national and international conferences and journals. His three papers received the Best Paper Award at several international conferences around the world. He received the Best Reviewer Award 2018 by ICT Expressed journal. Moreover, he received the Education and Research Award 2018 given by Bangladesh Community in Korea. He served as a reviewer for many international journals (including IEEE, Elsevier, Springer, ScienceDirect, and Hindawi published journals) and IEEE conferences. He has been working as an Editor for ICT Express, Associate Editor of IEEE Access, Lead Guest Editor for Wireless Communications and Mobile Computing, and Guest Editor for Applied Sciences. He was a TPC chair of the International Workshop on 5G/6G Mobile Communications in 2017 and 2018. He was the publicity chair of the International Conference on Artificial Intelligence in Information and Communication, 2019. He has served as a TPC member for several IEEE conferences. He has been involved in several Korean government projects. His research interests include convergence networks, QoS provisioning, small-cell networks, Internet of Things, eHealth, 5G and beyond (5GB) communications, and optical wireless communication. 


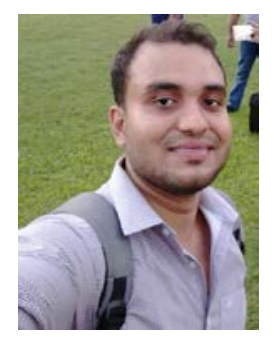

Md. Tashikur Rahman received the B.Sc. degree in Electrical and Electronic Engineering from Khulna University of Engineering and Technology, Bangladesh, in 2016. His research interests include optical wireless communications, optical camera communications, eHealth, artificial intelligence, 5G and beyond communications, game theory, heterogeneous networks.

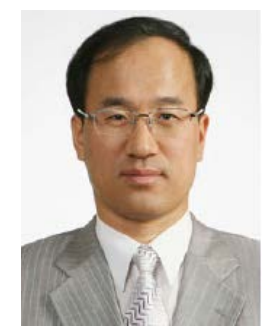

Yeong Min Jang received his B.E. and M.E. degrees in Electronics Engineering from Kyungpook National University, South Korea, in 1985 and 1987, respectively, and his Doctoral degree in computer science from the University of Massachusetts, USA, in 1999. He was with the Electronics and Telecommunications Research Institute (ETRI) from 1987 to 2000. Since 2002, he has been with the School of Electrical Engineering, Kookmin University, Seoul, South Korea, where he has been the Director of the Ubiquitous IT Convergence Center between 2005 and 2010, the Director of the LED Convergence Research Center since 2010, and the Director of the Internet of Energy Research Center since 2018. He is currently a life member of the Korean Institute of Communications and Information Sciences (KICS). He received the Young Science Award from the Korean Government (2003-2006). He has organized several conferences and workshops, such as the International Conference on Ubiquitous and Future Networks (2009-2017), the International Conference on ICT Convergence (2010-2016), the International Conference on Information Networking 2015, and the International Workshop on Optical Wireless LED Communication Networks (2013-2016). He had served as the Founding Chair of the KICS Technical Committee on Communication Networks in 2007 and 2008. He had served as the Executive Director of KICS from 2006 to 2014, Vice President of KICS from 2014 to 2016, and Executive Vice President of KICS for 2018. He is the president of KICS for 2019. He serves as the Co-Editor-in-Chief of ICT Express, which is published by Elsevier. He had been the Steering Chair of the Multi-Screen Service Forum since 2011 and the Society Safety System Forum since 2015. He had served as the Chairman of the IEEE 802.15 Optical Camera Communications Study Group in 2014 and also served as the Chairman of the IEEE $802.15 .7 m$ Optical Wireless Communications Task Group. He is currently the Chairman of IEEE 802.15 Vehicular Assistive Technology (VAT) Interest Group. His research interests include 5G/6G mobile communications, Internet of Energy, eHealth, multiscreen convergence, public safety, optical wireless communications, optical camera communication, and the Internet of Things. 